the Journal of Craniofacial Genetics and Developmental Biology, 1985, vol 5, is dedicated to the memory of Dr Samuel Pruzansky, an internationally renowned worker in the field of craniofacial malformations until his death in 1984. Among his many achievements, Dr Pruzansky organised the first international symposium on craniofacial malformations in 1959 and founded the first Center for Craniofacial Anomalies at the University of Illinois College of Medicine in 1967.

The book contains reviews and original papers by 48 contributors from six countries and covers a wide, though by no means complete, range of clinical and experimental topics. It is divided into sections I to VI. The first includes a brief account of the life and work of Dr Pruzansky and lists his more than 190 publications. Then follows an outline of his involvement with the Center for Craniofacial Anomalies, together with reviews of techniques, such as infant cephalometric radiography, that can be used in research and as aids to diagnosis and patient management. Section III deals with growth of the cleft palate during the first five years of life, with considerations that influence the timing of surgical closure, and with velopharyngeal inadequacy in the absence of overt cleft palate. It also contains a brief but intriguing chapter reporting an unusually high frequency of non-right handedness among parents of children with right sided cleft lip (the less frequently affected side). The largest section of the book, section IV, includes accounts of both cross sectional and longitudinal cephalometric studies of various disorders, among them a detailed analysis of achondroplasia. One chapter introduces a refreshingly simple system of six measurements, taken from cephalometric radiographs, as an aid to 'quantitative dysmorphology', perhaps a useful way of sidestepping the bewildering variety of angles and dimensions that have been used in the past. Section $\mathrm{V}$ contains a number of clinical observations and hypotheses to explain the pathogenesis of selected syndromes, together with a description of a computerised record system for craniofacial malformations and a discussion of chromosome damage and congenital defects associated with the infamous 'Agent Orange'. The final section is devoted to experimental animal studies, the most common theme being genotype-environment interaction in the aetiology of cleft lip and palate in mice. Also included is a report of experimental fusion of the naturally cleft embryonic chick palate through surgical intervention.

The book is not a medical genetics text and only occasionally is there mention of such aspects of the various syndromes as aetiological heterogeneity and genetic counselling. However, it does have a predominantly clinical orientation and so should find its widest readership among those, whatever their speciality, who work with patients having craniofacial malformations. It will also be of use to experimentalists striving towards a better understanding of the complexities of craniofacial development. The book is attractively produced, with reasonably clear line drawings and adequate half tone illustrations. Many of the chapters are likely to be of practical value to both clinicians and researchers, making the volume a fitting tribute to one whose professional life was devoted to helping patients with these disorders.

JEFFREY SOFAER

Sister Chromatid Exchanges. Part A. The Nature of SCEs. Part B. Genetic Toxicology and Human Studies

Edited by Raymond $\mathbf{R}$ Tice and Alexander Hollaender. (Pp 364; figures + tables. £58.00.) New York: Alan R Liss. 1985.

This treatise, consisting of two volumes, contains proceedings of the first ever international symposium on sister chromatid exchange (SCE), held at Brookhaven, NY in 1983, in which participants, largely through a series of research papers, provide an overview of this steadily expanding field.

Part $A$. The Nature of SCEs begins with a brief historical perspective. The existence of SCEs was initially postulated in 1938 by McClintock to account for the instability of ring chromosomes she had observed in maize. The subject then appears to have lain dormant until Taylor et al (1957) published the now famous technique of radiolabelling chromosomes with tritiated thymidine, which, due to the semiconservative nature of DNA replication, rendered SCEs visible at metaphase. This approach was given fresh impetus when Latt (1974) discovered an elegant method whereby bifiliary BrdU substituted DNA stained with a fluorochrome could be distinguished from unifiliary substituted DNA, allowing precise localisation of SCEs. Further refinement of the practicality of the technique by Perry and Wolff enabled SCEs to be readily observed in Giemsa stained preparations. The subsequent realisation that a large number of mutagens and clastogens were also inducers of SCEs has led to the method being accepted as a potentially sensitive assay for environmental genotoxic agents.

Contributors move on to consider the nature of SCE and explore the role of halogenated and natural pyrimidines, including the effects of thymidylate 
stress and DNA methylation. The mechanism of SCE induction, which is now generally believed to involve the replication fork, and its modulation through DNA repair, potentiating agents, and anticlastogens is also investigated. Characterisation of SCEs and the concomitant chromosomal aberrations is attempted and the interrelationship of SCEs, clastogenesis, mutagenesis, cracinogenesis, and environmental pollutants is examined in some detail. This volume concludes with papers on the statistical treatment of the subject.

Part B. Genetic Toxicology and Human Studies first addresses the use of SCE studies in investigating the effects of mutagens in vertebrate systems, both in vitro and in vivo; SCE persistence and the influence of cell cycle kinetics are examined in the human lymphocyte culture system in particular. The value of SCE studies in the diagnosis of inherited genetic disease and in the dosimetry of acquired genetic damage resulting from the use of various pharmaceutical products, especially cytostatic drugs, and environmental and occupational agents, such as cigarette smoke and styrene, is assessed: in this way the possibly premature concept of biomonitoring using SCE levels is tentatively explored. In addition, the inherent variability of spontaneous and induced SCE levels observed in man is evaluated as a possible factor in predisposition to cancer. The treatise ends with the summary of a general and apparently inconclusive discussion of the interpretation and predictive value of SCE data and its relevance to public health.

The fascinating phenomenon of SCE, despite recent advances, remains enigmatic with both its precise mechanism of induction and its clinical significance yet to be fully elucidated. Nevertheless, this treatise is a timely and very informative publication, which, considering the limitations imposed by the content, is quite well presented, the main deficiency being the lack of any meaningful synthesis of the mass of data reported. Overall, it is an excellent reference book and provides stimulating reading for researchers and those with a keen interest in cytogenetics, genetic toxicology, and related specialties.

\section{Atlas of Skeletal Dysplasias}

By R Wynne-Davies, C M Hall, and A G Apley. (Pp 600; figures + tables. $£ 90.00$.) Edinburgh: Churchill Livingstone. 1985.

This book is not the first to be devoted to bone dysplasias, but it is certainly quite different from any other that exists and stands in a class of its own. As its name implies, it is an atlas, but it is much more than this, being rather a detailed account of bone dysplasias as seen principally through their radiographic appearance.

This book starts with some useful introductory sections on normal $x$-ray appearances and prenatal detection, after which the major groups of primary bone dysplasias are dealt with successively and in detail. Wisely, the authors do not attempt to cover general malformations or limb defects except as they may affect bone dysplasias.

It is in the detailed treatment of the individual disorders that the book excels; $x$-rays are given in abundance to illustrate different manifestations at different ages, giving a much fuller and more balanced picture than is possible when only a few selected illustrations are allowed. Introducing each series of films is a brief but comprehensive text, which is particularly strong on natural history and complications, and almost always accurate regarding inheritance.

Inevitably in a volume of over 600 large pages, some faults can be found, but most are trivial; on genetics the only error I could find was the one widely repeated elsewhere of lethal osteogenesis imperfecta being recessively inherited. My only general criticism is that the standard of clinical photography is not always as high as that of the $x$-rays; care of background could have greatly improved some of these. The lack of gonadal shielding in a high proportion of $x$-rays is an indictment of much orthopaedic and radiological practice, but cannot be blamed on the authors!

Leaving aside these selected points the book as a whole is an outstanding achievement. It is thoroughly enjoyable to read, and is quite clearly the work of authors who are familiar with and experts on bone dysplasias as a whole, not just on their radiology or any other single aspect. It is this integrated approach that will give the book its real and lasting value. Despite its price it will surely prove an essential companion to anyone involved in the clinical and genetic management of this fascinating group of disorders. 